\title{
Pessaries for POP and SUI: Your options and guidance on use
}

\author{
Gynecologists may not always "think pessary first" when it comes to pelvic \\ organ prolapse management. However, it is important to be familiar with the \\ array of available pessary options and how to select a device based on the \\ patient's disorder and needs.
}

Henry M. Lerner, MD

\section{IN THIS} ARTICLE

Candidates for pessaries page 33

Pessary types page 34

History of pessaries page 36

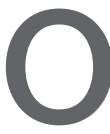

ver the last 30 years, surgical correction of the common condition pelvic organ prolapse (POP) and stress urinary incontinence (SUI) has become so routine and straightforward that many gynecologists and urogynecologists choose surgery as their first choice for treating these conditions, withholding it only from the riskiest patients or from those who, for a variety of reasons, do not choose surgery. Moreover, as generalist gynecologists increasingly refer patients with POP or incontinence to their urogynecologist colleagues, they increasingly lack the skills, or have not been trained, to use conservative treatment strategies for these disorders. Thus, pessaries-devices constructed of inert plastic, silicone, or latex and placed inside the vagina to support prolapsed pelvic structures-frequently are not part of the general gynecologist's armamentarium.

When properly selected, however, pessaries used for indicated purposes and correctly fitted are an excellent, inexpensive, lowrisk, and noninvasive tool that can provide

Dr. Lerner is Assistant Clinical Professor (retired), Harvard Medical School, Boston, Massachusetts.

The author reports no financial relationships relevant to this article.

doi: 10.12788 /obgm.0052 immediate relief not only of POP but also of SUI and defecatory difficulties. As an alternative to surgery, pessaries are especially valuable, because the other major nonsurgical modality for treatment of POP and incontinence-pelvic floor muscle training-often is not covered by insurance (making it expensive for patients), takes many weekly sessions to complete (which can make access challenging), and frequently is not readily available. ${ }^{1}$

POP is very common. An estimated $15 \%$ to $30 \%$ of women in North America have some degree of prolapse, and more than 500,000 surgeries for this condition are performed in the United States each year. ${ }^{2}$ Risk factors for POP include:

- vaginal childbirth, especially higher parity

- advancing age

- high body mass index (BMI)

- prior hysterectomy

- raised intra-abdominal pressure, such as from obesity, chronic cough, or heavy lifting.

In addition to the discomfort caused by the herniation of pelvic and vaginal structures, POP also is associated with urinary incontinence $(73 \%)$, urinary urgency and frequency (86\%), and fecal incontinence (31\%). ${ }^{3}$

Moreover, according to the US Census Bureau, the number of American women aged 65 or older will double to more than 40 million by $2030 .{ }^{4}$ This will greatly increase the 


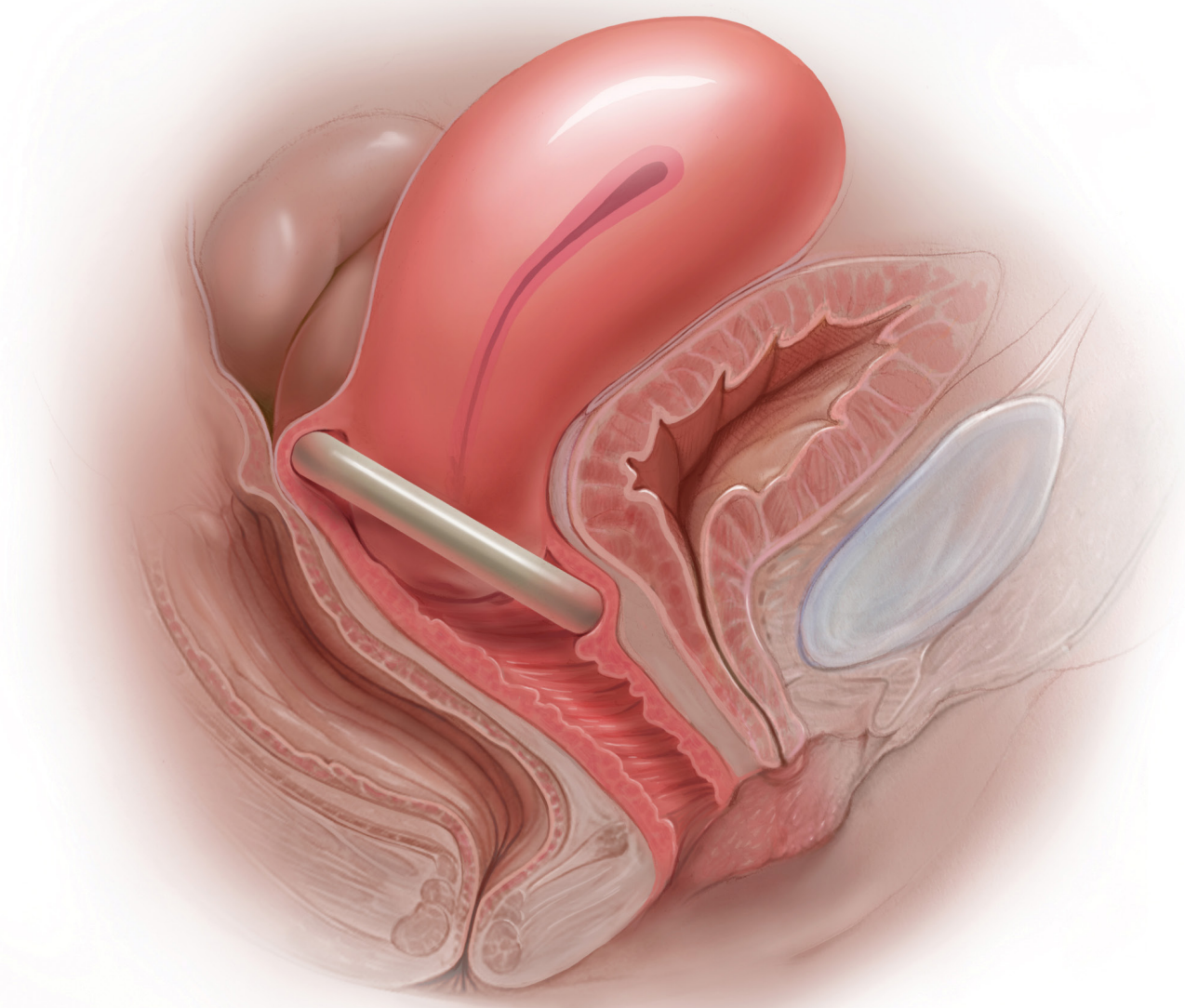

population of women at risk for POP who may be candidates for pessary use. It therefore behooves gynecologists to become familiar with the correct usage, fitting, and maintenance of this effective, nonsurgical mode of treatment for POP.

In this article, I discuss why pessaries are a good option for many patients with POP, review the types of pessaries available, and offer guidance on how to choose the right pessary for an individual patient's needs. In addition, the box on page 36 provides an interesting timeline of pessary history dating back to antiquity.

Next month in Part 2 of this article, I cover how to fit a pessary; device aftercare; potential complications of use; and effectiveness of pessaries for POP, SUI, preterm labor prevention, and defecatory disorders.

\section{Potential candidates for pessary use}

Almost all women with POP-and in many cases accompanying SUI-are potential candidates for a pessary. In fact, many urogynecologists believe that a trial of pessary usage should be the first treatment modality offered for POP. ${ }^{5}$ Women who cannot use a pessary include those with an extremely short vagina $(<6 \mathrm{~cm})$ and those who have severely eroded vaginal mucosa. In the latter situation, the mucosa can be treated with estrogen cream for several weeks and, once the tissue has healed, a pessary can be fitted.

Given that surgical repair is generally a straightforward, one-time procedure that obviates the need for long-term use of an artificial device worn internally, why might a patient or her physician opt for a pessary instead?

Some of the many reasons include:

- Many patients prefer to avoid surgery.

- Many patients are not appropriate candidates for surgery because they have significant comorbid risk factors or high BMI.

- Patients may have recurrent prolapse or incontinence and wish to avoid repeat surgery.

- Patients with SUI may have heard of the occurrence of mesh erosion and wish to avoid that possibility.

- Women who live in low-resource environments or countries where elective surgical 
care is relatively unavailable may not have the option of surgery.

A clinician might also recommend pessary use:

- as a diagnostic tool to attempt to assess the potential results of vaginal repair surgery

- to estimate the potential effectiveness of a midurethral sling procedure; several investigators have found this to be approximately as accurate as urodynamic testing ${ }^{6,7}$

- as prophylaxis for pregnant women with either a history of preterm cervical dilation or a short cervix detected on ultrasonography

- for pregnant women with POP that is worsening and becoming increasingly uncomfortable

- for women with POP who wish to have more children

- for short-term use while a patient is delaying or awaiting POP surgery or to allow time for other medical issues to resolve

- for patients who wish only intermittent, temporary support while exercising or engaging in sports.

\section{Patient acceptance may be contingent on counseling}

Numerous studies show that women who choose pessaries to treat POP are generally older than women who elect surgery. Still, patient acceptance of a trial of pessary use depends much on the counseling and information she receives. Properly informed, many patients with POP will opt for a trial of pessary placement. One study showed that, of women with untreated $\mathrm{POP}, 36 \%$ preferred pessary placement to surgery. ${ }^{8}$ Other investigators reported that when women with symptomatic POP had the benefits of a pessary versus surgery explained to them, nearly two-thirds opted for a pessary as their mode of treatment. ${ }^{9}$

\section{Exceptions to pessary use}

Fortunately, there are relatively few contraindications to pessary use. These are vaginal or pelvic infection and an exposed foreign body in the vagina, such as eroded vaginal mesh. In addition, patients at risk for nonadherence with follow-up care are poor candidates, as it could lead to missing such problems as mucosal erosion, ulceration, or even (extremely rarely) fistula formation. Pessaries may be inappropriate for sexually active women who on their own are unable to remove and reinsert pessary types that do not allow for intercourse while in place (see below).

\section{Types of pessaries}

The numerous kinds of pessaries available fall into 3 general categories: support, space filling, and lever, and devices within each group have modifications and variations. As with most areas of prescribing and treatment in medicine, it is best to become very familiar with just a few kinds of pessaries, know their indications, and use them when appropriate.

Most pessaries are constructed of inert silicone which, unlike earlier rubber pessaries, does not absorb odor or discharge. They are easy to clean, long lasting, and are autoclavable and hypoallergenic.

\section{Support pessaries}

Support pessaries look like contraceptive diaphragms. They are easy to place and remove, are comfortable, and do an excellent job correcting moderate POP. They also can control or eliminate symptoms of SUI by the pressure they exert on the urethra and their alteration of the urethrovesicular angle.

\section{Ring pessaries}

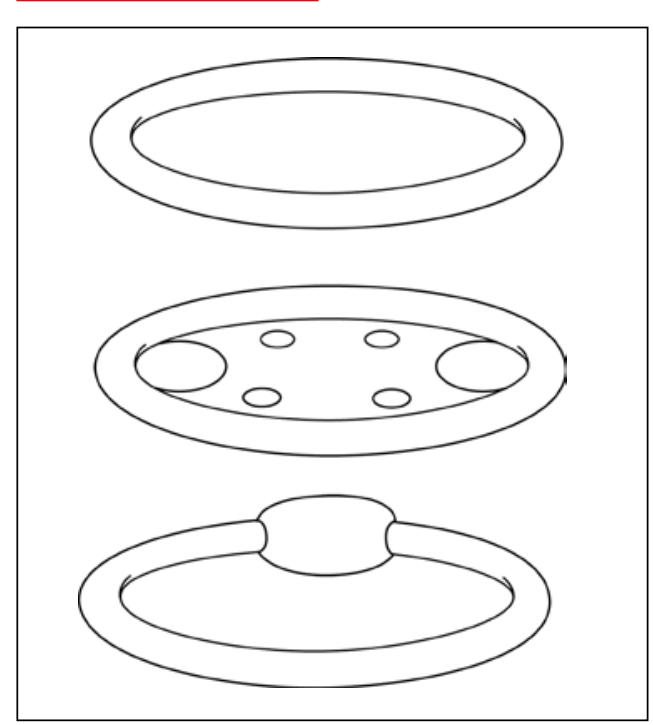




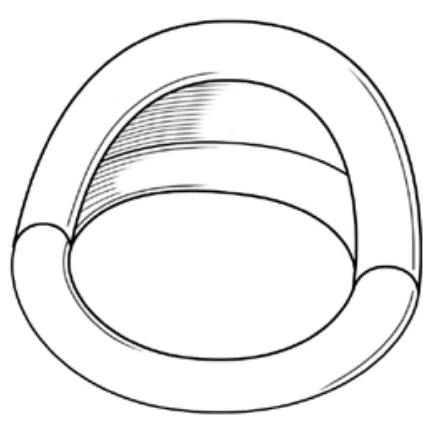

Ring pessaries. The most commonly used type of pessary, the ring pessary, ${ }^{10}$ comes in 4 variations:

- a simple open ring

- a ring with a web of material, called a "support shield," that fills the ring

- an open ring with a firm $2-\mathrm{cm}$ "incontinence knob" attached that is positioned over the urethra

- a ring with support shield and incontinence knob.

When in position, the deepest edge of a ring pessary fits behind the cervix (or in the vaginal apex for women who have had a hysterectomy) while the front of the ring slips into place behind the pubic symphysis, just like a diaphragm. When a ring with an incontinence knob is used, the ring is rotated until the knob is directly over the urethra.

Sexual intercourse is possible with any of the ring pessaries in place. Of the various types of pessaries, the ring pessary is the easiest to insert and remove. Some women tie a piece of dental floss to the edge of the ring to make its removal even easier.

The ring pessary is available in sizes $0(44.5 \mathrm{~mm})$ to $13(127 \mathrm{~mm})$. For most women a size 3,4 , or 5 ring pessary fits well.

The Marland pessary is similar to the ring pessary with the addition of a wedgeshaped piece of material approximately $3 \mathrm{~cm}$ in height that arises from half of the ring. It rarely is used in the United States because most American gynecologists are unfamiliar with it, and there is little evidence that it is more effective than the ring pessary. ${ }^{11}$
Shaatz pessary

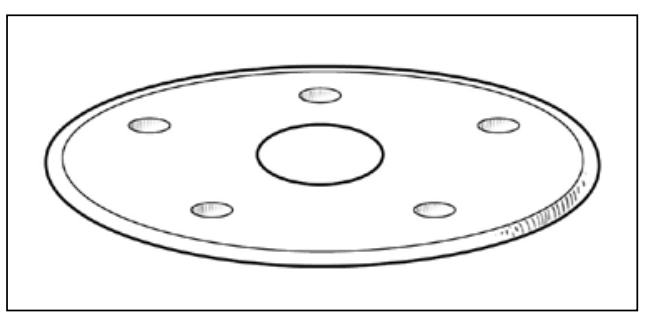

The Shaatz pessary is a rigid round pessary, smaller in diameter than the standard ring pessary, and similar to the Gellhorn pessary (discussed below) but without a stem. It is placed the same way one places a ring pessary but with its concave surface up against the cervix or, if there is no cervix, against the upper anterior vaginal wall. Its main benefit is that it provides firmer support than the ring pessary. This pessary is not widely used in the United States.

The Gehrung pessary looks like a flat strip of material that has been bent into the shape of a "U." It is designed to correct severe cystoceles and rectoceles. For insertion, the edges at the open end of the pessary are squeezed together and the pessary is inserted with the closed part of the " $U$ " facing the anterior vaginal wall. The upper edge is advanced until it rests in the anterior fornix of the vagina (or in the vaginal apex in women who have had a hysterectomy). Although it is more efficacious than some other pessaries for control of vaginal wall prolapse, its unfamiliarity to clinicians and its unusual shape result in it being used rarely.

\section{Gehrung pessary}

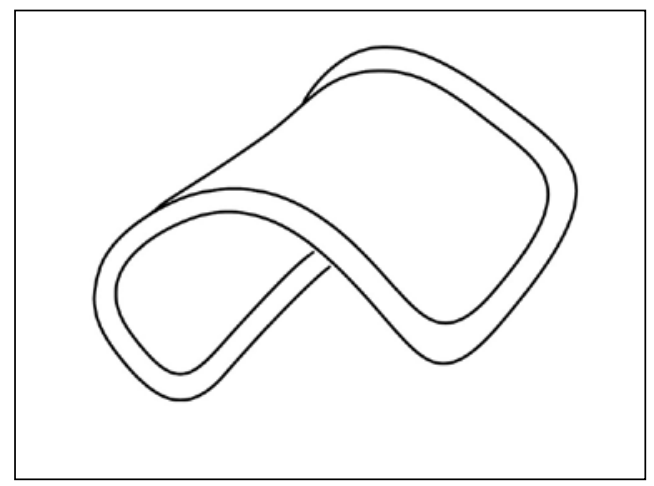




\section{Space-filling pessaries}

Space-filling pessaries are used when more severe degrees of prolapse are present than can be managed by the ring or other support pessaries. This is especially the case when the vagina is so capacious or the introitus so lax that a standard ring pessary cannot be kept in place, resulting in frequent expulsions.

Space-filling pessaries are 3 dimensional and work by filling the vagina with a relatively large object that prevents the cervix/vaginal apex from dropping down and the vaginal walls from prolapsing. They have a special role for women who:

- are posthysterectomy and have an enterocele and/or vaginal apex prolapse

- have significant rectoceles for which support pessaries are not effective
- have a wide vaginal hiatus and thus are prone to expel support pessaries.

Space-filling pessaries do have some drawbacks compared with support pessaries. For example, they do not help in controlling SUI, and they are difficult for patients to remove on their own for cleaning. In addition, sexual intercourse is impossible with a space-filling pessary in place.

The Gellhorn pessary is the most common of the space-filling pessaries, and it is the one gynecologists and urogynecologists most often use for severe prolapse. It has a concave disc that fits up against the cervix or vaginal apex and a solid stem that points down the vagina. The stem itself is supported by the perineal body. It offers excellent support for severe uterine and vaginal wall prolapse, as

\section{A brief history of pessaries}

Pessaries have been used in one form or another to help resolve pelvic organ prolapse (POP) in women for at least 2,500 years. They have come in many shapes and have been made of many materials. Here is a brief sketch of the history of the pessary.

\section{Antiquity}

Kahun papyrus (ancient Egypt, c. 2000 BCE)

Women with POP were made to stand over a fire in which different ingredients were burned. It was thought that the disagreeable odors emitted would cause the uterus to "rebel" and thus revert back into place. ${ }^{1}$

\section{Hippocrates (c. 460-375 BCE)}

Used several techniques to resolve uterine prolapse:

- Tipping the woman upside down and shaking her, using gravity as an aid to return the prolapsed organs into the pelvis ${ }^{2}$

- Cupping of the buttocks and the lower abdomen in hopes of "sucking" the prolapsed uterus back into place ${ }^{3}$

The Greek physician Polybus (c. 400 BCE) Placed half a pomegranate in the vagina to hold prolapsed structures in place $^{2}$

\section{Cleopatra (c. 70-30 BCE)}

Treated prolapse with the vaginal application of an astringent liquid ${ }^{2}$

Celsus (c. 25 BCE-50 CE)

Used cone-shaped pessaries made of bronze with a perforated circular plate on the lower edge through which bands were attached. The bands were then tied around the body to keep the device in place ${ }^{4}$

The Greek physician Soranus (c. 98-138) Utilized linen tampons soaked with vinegar - along with a piece of beef-to treat prolapse. These were then held in place by bands passed around the loins ${ }^{2}$

Galen (c. 130-210)

Used fumigation to "encourage" the uterus to return to the pelvis ${ }^{2}$

\section{Middle Ages}

Paulus of Aegina (c. 625-690) and

Abbas (c. 949-982)

Both wrote about the use of pessaries made of wax $^{3}$
Myrepsus (late 13th century)

Described the preparation of 45 types of pessaries consisting of different solid materials treated with perfumes, wax, honey, and herbs ${ }^{5}$

16th century

Caspar Stromayr (Practica Copiosa, 1559) Used as pessaries tightly rolled sponges bound with string, dipped in wax, and covered with oil or butter ${ }^{6}$

Ambroise Paré (c. 1510-1590)

Developed the first ring-type pessary in the late 16th century. He used hammered brass and waxed cork in the shape of an oval to treat uterine prolapse. He also made ring-shaped devices of gold, silver, or brass which were kept in place by a belt around the waist. ${ }^{7}$

\section{7th century}

de Castro (1546-1627)

Urged "attacking" uterine prolapse

with application of a red-hot iron thus "frightening it" into receding back into the vagina ${ }^{8}$ 
long as the perineal body is intact. The stem stabilizes the disc portion of the pessary and prevents pessary expulsion. Gellhorn pessaries are available with long or short stems.

The Gellhorn is inserted into the vagina by folding the stem 90 degrees until it is in the

\section{Gellhorn pessary}

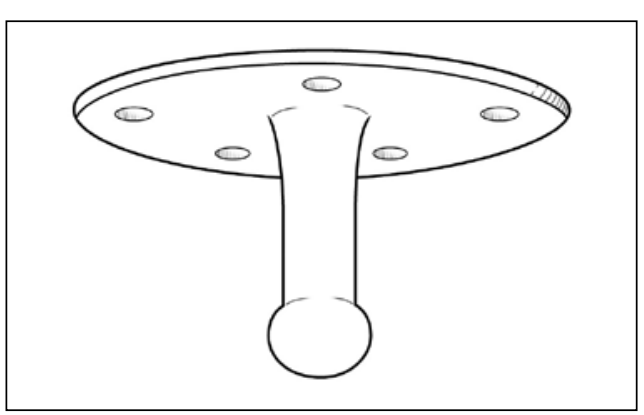

same plane as the disc. With lubricated fingers, the patient's perineal body is depressed and the disc of the pessary is folded and slid in. The disc is then placed up against the cervix or vaginal apex with the stem pointing down the vagina and tucked just inside the posterior edge of the introitus.

Removing the Gellhorn pessary can be problematic and is difficult for patients to do on their own. Clinicians often must use a ring forceps to grasp the stem of the pessary in order to bring it into the lower vagina, where the stem is folded up against the disc and the entire pessary removed. As with all space-filling pessaries, the Gellhorn must be taken out prior to intercourse.

The Gellhorn pessary is available in sizes that range from a disc diameter of 1.5 to
Hendrik van Roonhuyse (1625-1672)

In his gynecology textbook, discussed the etiology and treatment of prolapse. He utilized a cork with a hole in it (to allow for passage of discharge) as prolapse treatment. He also wrote of removing an obstructed wax pessary that had blocked discharge of a patient's vaginal secretions for many years ${ }^{4}$

\section{8th century}

Thomas Simson (1696-1764)

Invented a metal spring device that kept a pessary made of cork in place ${ }^{9}$

John Leake (1729-1792)

Recommended the use of sponges as pessaries to avoid vaginal prolapse ${ }^{10}$

\section{Juville (1783)}

Was the first to use rubber pessaries, resembling today's contraceptive cup, to avoid injuring the vaginal mucosa. The center of the cup was perforated with a gold tip which allowed for the discharge of vaginal secretions $^{10}$

\section{9th century \\ Scanzoni (1821-1891)}

Recommended massage and the application of leeches to reduce local congestion and swelling of prolapsed pelvic organs before manual replacement ${ }^{11}$
Hugh Lenox Hodge (1796-1873)

In his 1860 textbook Diseases

Peculiar to Women, Hodge discussed at length the use of pessaries for uterine displacement. He suggested that metals, alloys, glass, and porcelain be used for pessaries rather than cork, wax, and sponges ${ }^{12}$

\section{0th century} 1950s-

Pessaries made of rubber, which absorb discharge and odor, are replaced by polystyrene pessaries. Currently, pessaries are made of silicone, plastic, and latex.
References

1. Stevens JM. Gynecology from ancient Egypt: the papyrus Kahun, a translation of the oldest treatise on gynecology that has survived from the ancient world. Med J Austr. 1975;2:949-952.

2. Emge LA, Durfee RB. Pelvic organ prolapse: four thousand years of treatment. Clin Obstet Gynecol. 1966;9:997-1032.

3. Van Dongen L. The anatomy of genital prolapse. South Afr Med J. 1981;60:357-359.

4. Cianfrani T. Short History of Obstetrics and Gynecology. Springfield, IL: Charles C Thomas; 1960
5. Leonardo RA. History of Gynecology. New York, NY: Froben Press; 1944.

6. Tizzano AP, Muffly TM. Historical milestones in female pelvic surgery, gynecology, and female urology. In: Walters M, Karram M. Urogynecology and Reconstructive Pelvic Surgery, 4th ed. Philadelphia, PA: Elsevier Saunders; 2015

7. Farrell SA. Pessaries in Clinical Practice. Switzerland: Springer-Verlag; 2006.

8. Tam T, Davies MF, eds. Vaginal Pessaries. Boca Raton, FL: CRC Press; 2019.
9. Ricci JV. Genealogy of Gynaecology. Philadelphia, PA: Blakiston; 1950.

10. Miller DS. Contemporary use of the pessary. In Sciarra JJ, ed. Gynecology and Obstetrics. Philadelphia, PA: JB Lippincott Company; 1995.

11. Thomas TG. A Practical Treatise on the Disorders of Women. Philadelphia, PA: Lea Brothers and Co; 1891.

12. Hodge HL. Diseases Peculiar to Women, Including Displacements of the Uterus. Philadelphia, PA: Blanchard and Lea; 1860. 
FAST

TRACK

Because the donut pessary occupies a large space within the vagina, it is used for treatment of severe prolapse
Cube pessary

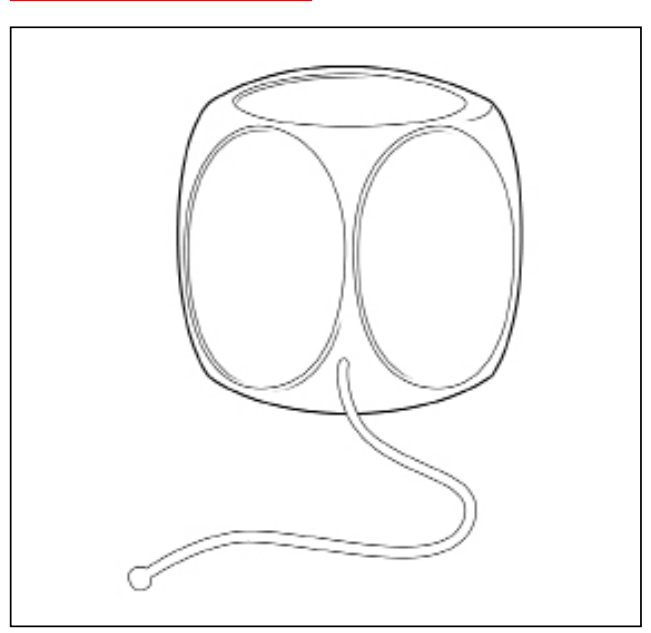

3.75 inches. Those measuring $2.5,2.75$, or 3 inches are used most commonly.

The cube pessary is a soft, dice-shaped piece of silicone with an indentation in each of its 6 sides. It is used for severe prolapse.

Squeezing the cube allows for easier insertion into the vagina; once it is at the top of the vagina, the cube expands back to its normal shape. The indentations on each side of the cube attach to the vaginal walls with moderate suction, which helps to keep the pessary in place. Because of the suction, the cube pessary can be used in cases of severe prolapse when other pessaries will not stay in place; a drawback is that the suction created by the indented sides can cause vaginal mucosal erosion. ${ }^{10}$ Ideally, the cube pessary should be removed every night for cleansing as discharge and accompanying odor can accumulate. The string attached to the cube pessary aids in its removal.

The cube pessary is available in sizes 0 to 7 , with edge lengths that range from 1 to 2.25 inches.

The donut pessary, as its name suggests, has the form of a large donut. It can be compressed slightly to help with insertion. Because it occupies a large space within the vagina, it is used (like the cube pessary) for treatment of severe prolapse. The size and shape of the donut pessary, however, can make it difficult for patients to insert and take out on their own.
Donut pessary

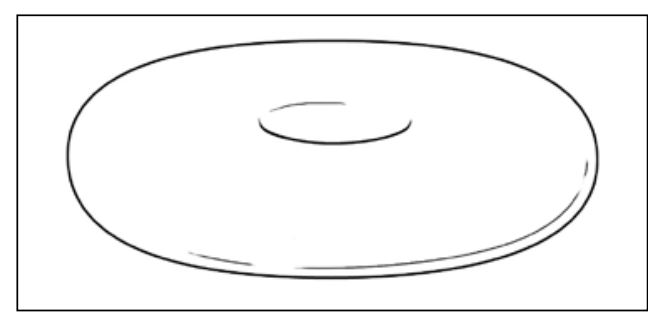

The donut pessary is available in sizes $0(51 \mathrm{~mm})$ to $8(95 \mathrm{~mm})$.

The inflatable pessary has the same basic shape as the donut pessary and serves the same purpose: It acts as a large semisoft object that fills the vagina to support the vaginal walls and cervix (or vaginal apex) in cases of severe prolapse. The inflatable pessary differs in that it has a valve on a stem through which air can be inserted and removed. This allows the uninflated pessary to be placed relatively easily into the vagina and then pumped full of air to the dimensions necessary to prevent vaginal, cervical, uterine, or apex prolapse. Air likewise can be removed to facilitate pessary removal.

One drawback of the inflatable pessary is that it is made of latex and thus cannot be used by anyone with a latex allergy. Also, as latex retains discharge and odors, this pessary should be removed and washed daily.

The inflatable pessary is available in sizes that range from 2 to 2.75 inches in 0.25 -inch increments.

Inflatable pessary

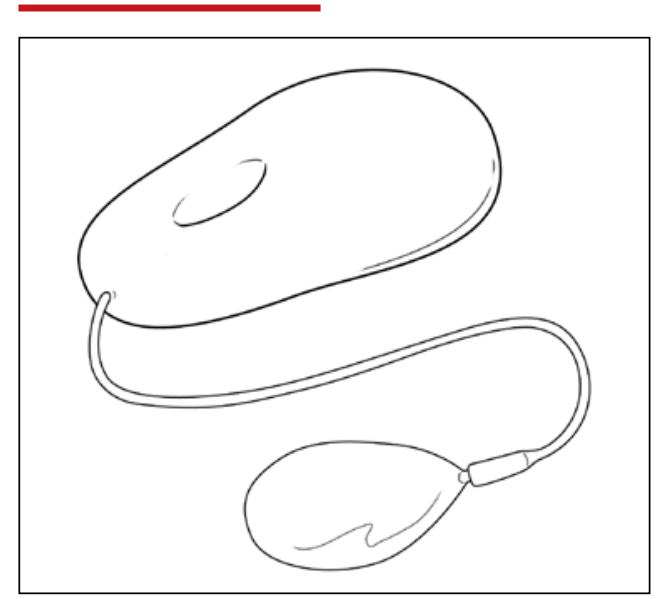


Lever pessary

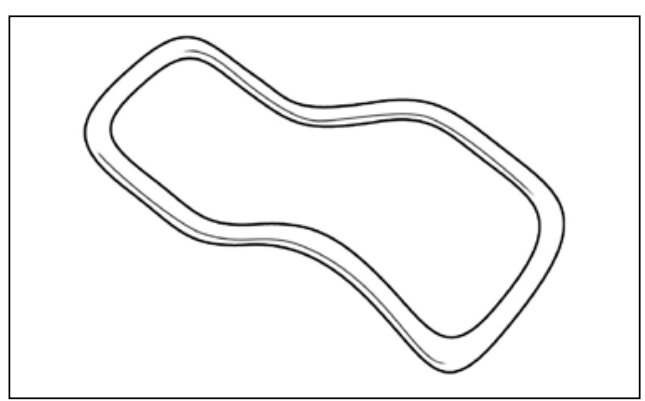

\section{Lever pessaries}

In addition to the more commonly used support and space-filling pessaries, there is a third kind that is rarely used in current practice: the lever pessaries. These pessariesthe Hodge, the Smith, and the Risser-are rectangles made of inert plastic that are folded into 3 planes to facilitate positioning in the vagina. The narrower of the 2 shorter ends of the folded rectangle is placed behind the cervix or at the vaginal apex while the other short end is placed behind the symphysis pubis.

Although sometimes used to correct POP in nonpregnant women, the lever pessary's main purpose is to antivert a retroflexed uterus and to support the cervix and uterus in cases of prolapse during pregnancy or impending cervical incompetence.

The 3 lever pessaries differ in terms of whether the narrow ends of the pessary are straight or curved and wider or narrower.

\section{How to choose the right pessary for your patient}

If a patient's POP or urinary incontinence symptoms would best be treated with a pessary, the next step is to select the pessary type and size best suited for that patient's needs and the size that should be prescribed. While there is controversy among experts as to whether or not certain pessaries are better than others for different indications, ${ }^{12}$ most gynecologists and urogynecologists who use pessaries on a regular basis agree on the following:

1. Support pessaries will meet the needs of most women with moderate POP and/or SUI. These include the ring pessary with or without the support shield and with or without an incontinence knob. A support pessary is the go-to pessary in most cases. Most women find it comfortable to wear, it is easy to put in and take out, and sexual intercourse is possible with the pessary in place.

2. The specific degree of a patient's prolapse and/or incontinence dictates whether or not to prescribe the support shield feature or the incontinence knob with a ring pessary. The shield helps support a prolapsed cervix and uterus when they are present. ${ }^{5,13}$ The knob is a useful feature if incontinence is a prominent symptom.

\section{The Gellhorn pessary is usually the} first choice for more severe prolapse. As long as there is some degree of posterior perineal support, this pessary does an excellent job of correcting even severe prolapse whether of a cervix and uterus or of vaginal walls and apex. It does require the patient to have some practice and dexterity for inserting and removing it on her own; individuals not comfortable or physically able to do so will need to have the pessary removed and cleaned by a clinician on a regular basis in the office. (Part 2 of this article will discuss pessary cleansing intervals).

\section{Space-filling pessaries (such as the} cube and donut) are useful when there is a severe degree of prolapse and insufficient perineal support to maintain a Gellhorn pessary. In practice, they are generally used less frequently-which is unfortunate, as they are a potentially useful solution for older women with severe prolapse who might not be candidates for surgical repair. As mentioned, both the cube and donut pessaries require more frequent removal for cleaning.

5. In unusual cases, the use of 2 pessaries simultaneously may resolve a difficult problem, such as when a pessary is the only option for treatment, the prolapse is severe, or it is impossible to find a pessary that resists being expelled from the vagina. ${ }^{14}$ A space-filling pessary in the most cephalad aspect of the vagina used in conjunction with a ring pessary with support shield below it can sometimes resolve even the worst cases of prolapse. 


\section{Pessaries}

CONTINUED FROM PAGE 39

\section{Stay tuned}

Part 2 of this article next month will provide more information on pessaries, including fitting, aftercare, potential complications, and effectiveness in various disorders.

\section{References}

1. Zoorob D, Higgins M, Swan K, et al. Barriers to pelvic floor physical therapy regarding treatment of high-tone pelvic floor dysfunction. Female Pelvic Med Reconstr Surg. 2017;23:444-448.

2. Kirby AC, Luber KM, Menefee SA. An update on the current and future demand for care of pelvic floor disorders in the United States. Am I Obstet Gynecol. 2013-209:584.el-584.e5.

3. Ellerkmann RM, Cundiff GW, Melick CF, et al. Correlation of symptoms with location and severity of pelvic organ prolapse. Am J Obstet Gynecol. 2001;185:1332-1337.

4. US Census Bureau. United States population projections: 2000 to 2050. https://www.census.gov/library/workingpapers/2009/demo/us-pop-proj-2000-2050.html. Accessed November 13, 2020.

5. Pott-Grinstein E, Newcomer JR. Gynecologists' patterns of prescribing pessaries. J Reprod Med. 2001;46:205-208.

6. Chaikin DC, Groutz A, Blaivas JG. Predicting the need for anti-incontinence surgery in continent women undergoing repair of severe urogenital prolapse. J Urol. 2000;163:531-534.

7. Reena C, Kekre AN, Kekre N. Occult stress incontinence in women with pelvic organ prolapse. Int J Gynaecol Obstet. 2007;97:31-34.
8. Thys SD, Roovers JP, Geomini PM, et al. Do patients prefer a pessary or surgery as primary treatment for pelvic organ prolapse. Gynecol Obstet Invest. 2012;74:6-12.

9. Kapoor DS, Thakar R, Sultan AH, et al. Conservative versus surgical management of prolapse: what dictates patient choice? Int Urogynecol J Pelvic Floor Dysfunct. 2009;20: 1157-1161.

10. Wu V, Farrel SA, Baskett TF, et al. A simplified protocol for pessary management. Obstet Gynecol. 1997;90:990-994.

11. Culligan PJ. Nonsurgical management of pelvic organ prolapse. Obstet Gynecol. 2012;119:852-860.

12. Cundiff GW, Amundsen CL, Bent AE, et al. The PESSRI study: symptom relief outcomes of a randomized crossover trial of the ring and Gellhorn pessaries. Am J Obstet Gynecol. 2007;196:405.el-404.e8.

13. Cundiff GW, Weidner AC, Visco AG, et al. A survey of pessary use by members of the American Urogynecologic Society. Obstet Gynecol. 2000;95(6 pt 1):931-935.

14. Singh K, Reid W. Nonsurgical treatment of uterovaginal prolapse using double vaginal rings. Br J Obstet Gynecol. 2001;108:112-113. 